\title{
Modelagem do comportamento dispersivo de material impactante em um lago por meio da Equação de Difusão-Advecção
}

\author{
Modeling of the dispersive behavior of impacting material in a lake by \\ Diffusion-Advection Equation
}

\author{
Arinos Alves da Silva Lopes, Jessica Lemos Gomes, Lays Carolline Souza e
}

\author{
Jaqueline Maria da Silva \\ Universidade Federal dos Vales do Jequitinhonha e Mucuri, Brasil \\ arinos_alves@hotmail.com; jelemos12@hotmail.com; lays.c.souza@hotmail.com; jaqueline.silva@ufvjm.edu.br
}

\begin{abstract}
Resumo
O crescimento exponencial e desenfreado da população mundial pode gerar problemas para a natureza e a saúde e qualidade de vida da população, tais como o desmatamento para abrir espaço para novas moradias, maior demanda de abastecimento de água e o aumento na quantidade de cargas poluentes lançadas em corpos hídricos. Diversos modelos matemáticos foram desenvolvidos a fim de tentar compreender e solucionar problemas de dispersão de poluentes em meios aquáticos. O objetivo desse trabalho é estudar, analisar e modelar o comportamento da dispersão de contaminantes e poluentes em um lago do município de Teófilo Otoni utilizando o modelo de equações diferenciais de Difusão-Advecção.

Palavras-chave: Difusão-Advecção, Método de Diferenças Finitas, Fluidos, Contaminação, Águas rasas, Discretização.
\end{abstract}

\begin{abstract}
The exponential and uncontrolled growth of the world population can affect the population's quality of life and lead to various environment and health problems, such as deforestation to make way for new housing and an increase in the water supply demand and in the amount of pollutants released in the water bodies. Several mathematical models have been developed to try to understand and solve problems of dispersion of pollutants in aquatic environments. This article aims to study, analyze and model the dispersion behavior of contaminants and pollutants in a lake using the Diffusion-Advection differential equations model. Keywords: Diffusion-Advection, Finite Difference Method, Fluids, Contamination, Shallow waters, Discretization.
\end{abstract}




\section{Introdução}

O crescimento da população mundial vem acontecendo de forma exponencial e desenfreada. Segundo dados do Centro Regional de Informação das Nações Unidas, $54 \%$ da população mundial vive em áreas urbanas e estima-se que em 2050 esse número aumente para $66 \%$. Este aumento significa que o crescimento populacional poderá trazer mais de 2,5 milhões de pessoas para as áreas urbanas em 2050 (UNRIC, 2014).

O crescimento populacional unido à urbanização cria diversos problemas para a natureza, saúde da população e qualidade de vida, tais como o desmatamento motivado pela abertura de novas áreas para a indústria e moradia, maior demanda de abastecimento de água para a população e, principalmente, o crescimento nos níveis de cargas poluentes em corpos hídricos, seja por cargas industriais ou domésticas. Estudos apontam que entre os 500 maiores rios do mundo, mais da metade enfrentam graves problemas de poluição (Abril, 2014).

Segundo (USP, 2014), 25 milhões de pessoas no mundo morrem por ano devido a doenças transmitidas pela água, como cólera e diarréias. Cerca de 50\% do volume da água utilizada no Brasil é proveniente de rios e $30 \%$ advém de lagos, lagoas e açudes e em grande parte a qualidade da água produzida não atende ao padrão de potabilidade vigente no país. Em países em desenvolvimento como o Brasil, cerca de $70 \%$ da população rural e $25 \%$ da população urbana não dispõem de abastecimento adequado de água potável.

Visando compreender e encontrar possíveis soluções para os problemas de dispersão de poluentes em meios aquáticos, a modelagem matemática se destaca como ferramenta científica fundamental neste processo de construção do conhecimento (Bassanezi, 2002).

Diversos modelos matemáticos foram desenvolvidos ao longo dos anos. O primeiro modelo que surge na literatura foi proposto por Streeter-Phelps que fornecia uma visualização do comportamento do corpo d'água em relação à quantidade de oxigênio dissolvido ao longo do seu curso ou do tempo após a recepção do esgoto. Despois dele vieram outros modelos como: o QUAL2E, que avalia o impacto do lançamento de cargas poluentes na água em estado estacionário e determina os efeitos da variação de dados meteorológicos e mudanças de concentração de oxigênio dissolvido no estado dinâmico; o MIKE11, que simula as variações nas descargas e no nível da água em rios como resultado da precipitação ocorrida na bacia hidrográfica, e as entradas e saídas das condições de contorno do corpo d'água; e o modelo de difusão-advecção, que permite descrever o transporte, a dispersão e a decomposição de substâncias dissolvidas ou suspenças (Vargas et al., 2001; Fleck et al., 2013). Por apresentar tais características, foi escolhido como ferramenta para dar início aos estudos apresentados neste trabalho.

\section{O modelo de Difusão-Advecção}

Nesta seção apresentamos o modelo de difusão-advecção usado neste trabalho para analisar a dispersão de um poluente num lago localizado no Bairro Marajoara no município de Teófilo Otoni, no nordeste do estado de Minas Gerais.

Desta forma, considere hipoteticamente $C(t, x, y)$ a concentração de um poluente residencial no instante $t \in I(0, t]$ em um ponto $(x, y) \in \Omega$ e seja $\Omega \subset \mathbb{R}^{2}$ o domínio da função concentração na superfície do lago.

Incorporando os modelos clássicos de Okubo (1980) onde difusão $=\operatorname{div}(\alpha \nabla C)$, de Edelstein-Keshet (1988) sendo o transporte advectivo div(V.C) e de Marchuk (1986) onde o fator de decaimento é $\sigma C$, em conjunto com uma fonte poluidora constante, obtém-se o modelo de difusão-advecção. Então, o problema proposto pode ser apresentado na forma $C(t, x, y)=$ difusão + transporte - decaimento + fonte (Prestes et al., 2013; Poletti, 2009). Matematicamente, tem-se:

$$
\frac{\partial C}{\partial t}=\operatorname{div}(\alpha \nabla C)-\operatorname{div}(\mathbb{V} . C)-\sigma C+f, t \in I e(x, y) \in \Omega .
$$

Na equação acima $\alpha$ é o coeficiente de difusibilidade constante do poluente na região $\Omega$, sendo calculado por $\alpha=\frac{\text { área }}{\text { tempo }} ; \sigma$ é o decaimento do poluente que varia linearmente com a presença do poluente $C(t, x, y) ; \mathbb{V}$ é o campo vetorial de velocidade, sendo esse constante e com divergente nulo e $f$ a fonte local (pontual) por onde ocorre o ingresso do poluente no lago. Desta forma:

$$
\frac{\partial C}{\partial t}=\alpha \nabla^{2} C-\mathbb{V} \nabla C-\sigma C+f, t \in I e(x, y) \in \Omega .
$$

O fenômeno da difusão representa o espalhamento natural do poluente no meio aquático devido a movimentos moleculares aleatórios ou de efeitos de turbulência. $\mathrm{O}$ transporte advectivo é provocado por agentes externos como vento, correnteza, movimentação de partículas e outros. O decaimento é o fenômeno que reúne alterações sofridas pelas moléculas do contaminante que reage com o meio externo ao longo do tempo, ocasionando perda de massa, e portanto, sua exclusão do meio (Danconi et al., 2013).

\subsection{Discretizações do modelo para uma ma- lha retangular}

Para que a solução de uma equação diferencial parcial se aproxime, de alguma forma, da solução real deve-se 
observar a consistência das equações de diferenças finitas e a estabilidade e convergência do método numérico empregado. Portanto, é necessário definir um critério de convergência que não amplifique os erros que aparecem durante o processo de simulação numérica. Para verificar a estabilidade da simulação, o presente trabalho utilizou o método de Von Neuman, sendo este um dos métodos mais empregadas no estudo da estabilidade de equações de diferenças finitas (Fortuna, 2000).

No contorno da região do Lago Marajoara utilizouse a condição de contorno de Von Neumann obtida da expressão geral (Júnior e Schulz, 2012):

$$
\alpha \frac{\partial C}{\partial \eta}+b C=h, \text { para }(x, y) \in \partial \Omega \text { e } t \in I .
$$

Na equação acima $\eta$ representa a normal unitária exterior à fronteira. As condições de contorno do problema foram estabelecidas considerando que não há fluxo no contorno. Portanto, na fronteira adotamos $\alpha=1, b=h=0$.

Em casos de transporte de contaminantes em meios aquáticos, para a resolução do problema com o modelo de difusão-advecção, é necessária a utilização de métodos numéricos capazes de utilizar malhas nãoestruturadas, como o Método de Diferenças Finitas (MDF) e o Método de Crank-Nicolson (MCK). Portanto, para que o modelo seja melhor analisado, é necessário discretizálo.

A primeira discretização apresentada neste estudo é a discretização espacial, considerando $t$ fixo e observando somente a dispersão da carga poluente no espaço. Para este tipo de discretização usou-se o MDF. A segunda discretização realizada foi a discretização temporal. Nesse caso, é necessário fixar um ponto $(x, y)$ e analisar o comportamento do material impactante no meio para $t \in I(0, t]$. Para esta discretização usou-se o MCK (Prestes et al., 2013).

\subsubsection{Discretização espacial e temporal}

$$
\begin{gathered}
\frac{\partial C^{2}}{\partial x^{2}}\left(x_{i}, y_{j}, t_{n}\right) \cong \frac{C_{i-1, j}^{n}-2 C_{i, j}^{n}+C_{i+1, j}^{n}}{\Delta x^{2}} \\
\frac{\partial C}{\partial x}\left(x_{i}, y_{j}, t_{n}\right) \cong \frac{C_{i+1, j}^{n}-C_{i-1, j}^{n}}{2 \Delta x} \\
\frac{\partial C^{2}}{\partial y^{2}}\left(x_{i}, y_{j}, t_{n}\right) \cong \frac{C_{i, j-1}^{n}-2 C_{i, j}^{n}+C_{i, j+1}^{n}}{\Delta y^{2}} \\
\frac{\partial C}{\partial y}\left(x_{i}, y_{j}, t_{n}\right) \cong \frac{C_{i, j+1}^{n}-C_{i, j-1}^{n}}{2 \Delta x}
\end{gathered}
$$

$$
\frac{\partial C}{\partial t}\left(x_{i}, y_{j}, t_{n+\frac{\Delta t}{2}}\right) \cong \frac{C_{i, j}^{n}-C_{i, j}^{n}}{\Delta t}+0\left(\Delta t^{2}\right) .
$$

Para tal aproximação, estima-se que

$$
c_{i, j}^{n+1}=C\left(x_{i}, y_{j}, t_{\left(n+\frac{\Delta t}{2}\right)}\right) .
$$

Desta forma chega-se a seguinte equação:

$$
C^{n+\frac{1}{2} i, j} \cong \frac{C_{i, j}^{n}+C_{i, j}^{n+1}}{2}=C_{i, j}^{n+\frac{1}{2}}+0\left(\Delta t^{2}\right) .
$$

Combinando as equações (1), (2) a (5) e (6) obtemos a discretização dos pontos interiores do domínio de $\Omega$ que é dada por:

$$
\begin{array}{r}
C_{i-1, j}^{n+1}\left(\frac{-\alpha \Delta t}{2 \Delta x^{2}}\right)-C_{i-1, j}^{n+1}\left(\frac{u \Delta t}{4 \Delta x}\right)-C_{i, j-1}^{n+1}\left(\frac{\alpha \Delta t}{2 \Delta y^{2}}\right) \\
-C_{i, j-1}^{n+1}\left(\frac{v \Delta t}{4 \Delta y}\right)+C_{i, j}^{n+1}+C_{i, j}^{n+1}\left(\frac{\alpha \Delta t}{\Delta x^{2}}\right) \\
+C_{i, j}^{n+1}\left(\frac{\alpha \Delta t}{\Delta y^{2}}\right)+C_{i, j}^{n+1}\left(\frac{\sigma \Delta t}{2}\right)-C_{i, j+1}^{n+1}\left(\frac{\alpha \Delta t}{2 \Delta y^{2}}\right) \\
+C_{i, j+1}^{n+1}\left(\frac{v \Delta t}{4 \Delta y}\right)-C_{i+1, j}^{n+1}\left(\frac{\alpha \Delta t}{2 \Delta x^{2}}\right)+C_{i+1, j}^{n+1}\left(\frac{u \Delta t}{4 \Delta x}\right) \\
=C_{i-1, j}^{n}\left(\frac{-\alpha \Delta t}{2 \Delta x^{2}}\right)+C_{i-1, j}^{n}\left(\frac{u \Delta t}{4 \Delta x}\right)+C_{i, j-1}^{n}\left(\frac{\alpha \Delta t}{2 \Delta y^{2}}\right) \\
+C_{i, j-1}^{n}\left(\frac{v \Delta t}{4 \Delta y}\right)+C_{i, j}^{n}-C_{i, j}^{n}\left(\frac{\alpha \Delta t}{\Delta x^{2}}\right)-C_{i, j}^{n}\left(\frac{\alpha \Delta t}{\Delta y^{2}}\right) \\
-C_{i, j}^{n}\left(\frac{\sigma \Delta t}{2}\right)+C_{i, j+1}^{n}\left(\frac{\alpha \Delta t}{2 \Delta y^{2}}\right)-C_{i, j+1}^{n}\left(\frac{v \Delta t}{4 \Delta y}\right) \\
+C_{i+1, j}^{n}\left(\frac{\alpha \Delta t}{2 \Delta x^{2}}\right)-C_{i+1, j}^{n}\left(\frac{u \Delta t}{4 \Delta x}\right)+\Delta t f_{i, j} .
\end{array}
$$

Pelas considerações feitas para a condição de contorno na fronteira temos a seguinte expressão $\frac{\partial C}{\partial \eta}=0 \in$ $\partial \Omega$, denominada de condição de Von Neumann homogênia. Nas fronteiras horizontais, tem-se $\frac{\partial C}{\partial \eta}= \pm \frac{\partial C}{\partial y} \mathrm{e}$ nas fronteiras verticais $\frac{\partial C}{\partial \eta}= \pm \frac{\partial C}{\partial x}$.

Considerando uma fronteira horizontal em que o nó $C_{i, j+1}$ (ponto virtual) não esteja no domínio da função, sendo este nó virtual e denominado como $C_{\nexists}$, e utilizando a equação (5) temos:

$$
\frac{\partial C}{\partial y}=\frac{C_{\nexists}^{n}-C_{i, j-1}^{n}}{2 \Delta y}=0 .
$$

Portanto $C_{\nexists}^{n}=C_{i, j-1}^{n}$. Substituindo (5) em (4), temos:

$$
\frac{\partial C^{2}}{\partial y^{2}}=\frac{2 C_{i, j}^{n}-C_{i, j}^{n}}{\Delta y^{2}}
$$


Para casos com apenas dois nós no domínio, o raciocíonio é análogo, porém têm-se dois pontos virtuais, $C_{\nexists x}$ e $C_{\nexists y}$. Neste caso, a equação se escreve como:

$$
\begin{array}{r}
C_{i, j}^{n+1}+C_{i, j}^{n+1}\left(\frac{\alpha \Delta t}{\Delta x^{2}}\right)+C_{i, j}^{n+1}\left(\frac{\alpha \Delta t}{\Delta y^{2}}\right) \\
+C_{i, j}^{n+1}\left(\frac{\sigma \Delta t}{2}\right)-C_{i+1, j}^{n+1}\left(\frac{\alpha \Delta t}{\Delta x^{2}}\right)-C_{i, j+1}^{n+1}\left(\frac{\alpha \Delta t}{\Delta y^{2}}\right) \\
=C_{i, j}^{n}-C_{i, j}^{n}\left(\frac{\alpha \Delta t}{\Delta x^{2}}\right)-c_{i, j}^{n}\left(\frac{\alpha \Delta t}{\Delta y^{2}}\right)+C_{i, j}^{n}\left(\frac{\sigma \Delta t}{2}\right) \\
+C_{i+1, j}^{n}\left(\frac{\alpha \Delta t}{\Delta x^{2}}\right)+C_{i, j+1}^{n}\left(\frac{\alpha \Delta t}{\Delta y^{2}}\right)+\Delta t f_{i, j} .
\end{array}
$$

\section{Um estudo de caso - O Lago Mara- joara}

O lago objeto deste estudo fica localizado no bairro do Marajoara, situado no município de Teófilo Otoni, na região nordeste do estado de Minas Gerais. O bairro é bastante frequentado pois, além de ser em grande parte residencial, ainda possui vários estabelecimentos de serviços médicos, centros educacionais, restaurantes, igrejas, setores de prestações de serviços públicos e um hospital público.

Neste sentido, este trabalho apresenta um estudo de extrema importância para auxiliar os órgãos gestores a propor a inserção de políticas públicas que visem a melhora da qualidade de vida da população que reside no bairro do Marajoara e daqueles que por lá circulam. Além disso, visa tentar proporcionar um ambiente de lazer menos nocivo e mais agradável, buscando a preservação desse bem tão importante e com risco de escassez, a água.

\subsection{Metodologia}

Para a resolução do modelo proposto, faz-se necessário a criação de uma malha do domínio. Neste trabalho usou-se o aplicativo Gmsh para a construção da malha, cuja topologia empregada foi a retangular.

Além disso, através do software Google Earth gerouse a imagem do lago situado na cidade de Teófilo Otoni MG, bairro Marajoara (Figura 1) (Figura 2). Tal imagem foi importada para o $\mathrm{Gmsh}$, que foi usado para delimitar as margens do lago, traçar o plano do domínio e gerar a malha retangular apresentada na Figura 3. Este procedimento pode também ser verificado em (Lopes et al., 2014).

O valor da velocidade dos ventos e de suas direções foram obtidos através do cálculo das médias das velocidades disponibilizadas pelo Instituto Nacional de Meteorologia (INMET, 2015). As medições utilizadas foram coletadas no período de 16 de janeiro de 2015 a 05 de fevereiro de 2015 . O ponto de coleta utilizado

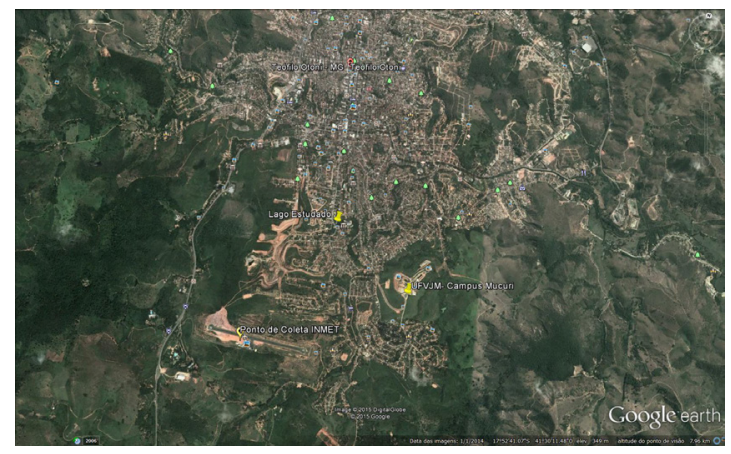

Figura 1: Localização do lago Marajoara na cidade de Teófilo Otoni - MG, obtidas pelo aplicativo Google Earth.

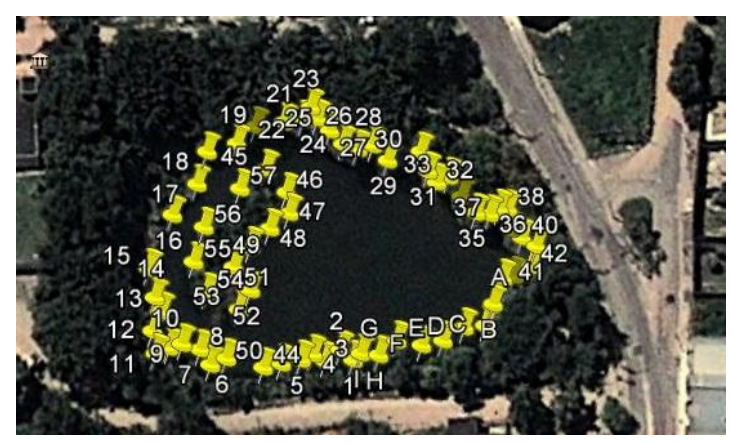

Figura 2: Imagem aproximada do lago Marajoara na cidade de Teófilo Otoni - MG, obtidas pelo aplicativo Google Earth.

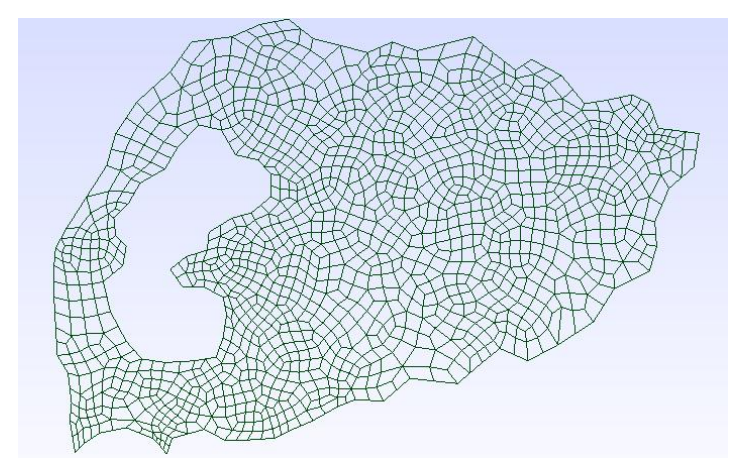

Figura 3: Malha retangular do lago Marajoara na cidade de Teófilo Otoni - MG, obtidas pelo aplicativo GMSH.

foi o aeroporto da cidade de Teófilo Otoni - MG que se encontra a 468 metros acima do nível do mar, como mostra a Figura 4.

A fonte pontual por onde ocorre o ingresso do poluente no lago está localizado próximo a margem superior esquerda.

Os dados coletados do INMET estão apresentados 


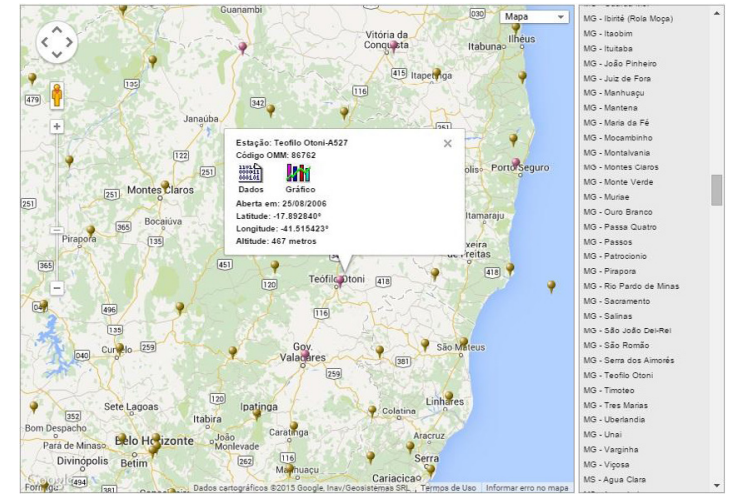

Figura 4: Dados de coleta de velocidade do vento (INMET, 2015).

na Tabela 1. Observa-se que a direção predominante dos ventos no município é para nordeste e faz-se a média das velocidades $V_{\text {vento }}=8,64 \mathrm{~km} / \mathrm{h}$ e das direções $\theta=77,59^{\circ}$. Para simplificar os estudos, neste modelo a velocidade e a direção do vento serão consideradas constantes em todo domínio.

O vetor da velocidade dos ventos $\left(V_{v}\right)$ atuantes na superfície do lago foi considerado como sendo 3\% de $V_{\text {vento }}$. Usando a aproximação linear clássica da Equação de Ekman, temos:

$$
V_{v}=0,03 \times 8,64=0,2592 \mathrm{~km} / \mathrm{h} .
$$

Para efeito de cálculo faz-se a decomposição do vento da velocidade dos ventos, chegando nos seguintes resultados:

$$
\begin{aligned}
& u=0,2531 \mathrm{~km} / \mathrm{h} \\
& v=0,0557 \mathrm{~km} / \mathrm{h} .
\end{aligned}
$$

O intervalo de tempo em horas utilizado para o estudo foi $(0, T]$ com $0<\mathrm{T} \leqslant 480$, referente à 20 dias.

A difusibilidade adotada será:

$$
\alpha=\frac{3,09 \times 10^{-3}}{480}=8,143 \times 10^{-6} \frac{\mathrm{km}^{2}}{\mathrm{~h}} .
$$

O decaimanto será:

$$
\sigma=2,083 \times 10^{-3} h^{-1} \text {. }
$$

O valor utilizado para o decaimento foi adotado hipoteticamente considerando-se o limite superior do intervalo de tempo, que é de 480 horas, e que se refere ao período em que aconteceram as medições dos ventos.

De acordo com os resultados obtidos através da discretização da área do lago vamos usar as aproximações de $\Delta x=0,0977 \mathrm{~km}$ e $\Delta y=0,0977 \mathrm{~km}$.
Tabela 1: Velocidade do vento, medido no aeroporto da cidade de Teófilo Otoni - MG, obtido pelo (INMET, 2015).

\begin{tabular}{|c|c|c|c|c|}
\hline Data & $\mathrm{m} / \mathrm{s}$ & $\mathrm{km} / \mathrm{h}$ & Direção & Graus \\
\hline $16 / 01 / 2015$ & 2,62 & 9,44 & $\mathrm{NE}$ & 76,63 \\
\hline $17 / 01 / 2015$ & 1,73 & 6,21 & $\mathrm{NE}$ & 63,54 \\
\hline $18 / 01 / 2015$ & 2,48 & 8,92 & $\mathrm{NE}$ & 78,08 \\
\hline $19 / 01 / 2015$ & 2,52 & 9,08 & $\mathrm{NE}$ & 68,17 \\
\hline $20 / 01 / 2015$ & 2,63 & 9,48 & $\mathrm{NE}$ & 72,00 \\
\hline $21 / 01 / 2015$ & 2,16 & 7,79 & $\mathrm{NE}$ & 84,04 \\
\hline $22 / 01 / 2015$ & 2,50 & 9,00 & $\mathrm{NE}$ & 70,13 \\
\hline $23 / 01 / 2015$ & 3,08 & 11,07 & $\mathrm{NE}$ & 74,83 \\
\hline $24 / 01 / 2015$ & 3,20 & 11,52 & $\mathrm{NE}$ & 74,33 \\
\hline $25 / 01 / 2015$ & 2,83 & 10,17 & $\mathrm{NE}$ & 71,17 \\
\hline $26 / 01 / 2015$ & 1,78 & 6,41 & $\mathrm{NE}$ & 66,38 \\
\hline $27 / 01 / 2015$ & 2,45 & 8,84 & $\mathrm{NE}$ & 79,75 \\
\hline $28 / 01 / 2015$ & 2,68 & 9,66 & $\mathrm{NE}$ & 73,38 \\
\hline $29 / 01 / 2015$ & 2,30 & 8,27 & $\mathrm{NE}$ & 71,42 \\
\hline $30 / 01 / 2015$ & 1,67 & 6,00 & $\mathrm{NE}$ & 75,67 \\
\hline $31 / 01 / 2015$ & 1,78 & 6,39 & $\mathrm{NE}$ & 89,08 \\
\hline $01 / 02 / 2015$ & 2,94 & 10,58 & $\mathrm{NE}$ & 83,50 \\
\hline $02 / 02 / 2015$ & 2,40 & 8,66 & $\mathrm{NE}$ & 71,00 \\
\hline $03 / 02 / 2015$ & 2,71 & 9,75 & $\mathrm{NE}$ & 81,17 \\
\hline $04 / 02 / 2015$ & 2,31 & 8,31 & $\mathrm{NE}$ & 74,25 \\
\hline $05 / 02 / 2015$ & 1,64 & 5,89 & $\mathrm{SE}$ & 130,84 \\
\hline
\end{tabular}

\subsection{Resultados Obtidos}

Com os parâmetros obtidos, calculados e apresentados neste estudo, apresenta-se os resultados das simulações realizadas no software Matlab: Figura 5, Figura 6, Figura 7, Figura 8, Figura 9, Figura 10.

Para critério de verificação foram realizadas simulações com os parâmetros de decaimento e difusibilidade obtidos em (Prestes et al., 2013), como pode ser observado em: Figura 11, Figura 12, Figura 13, Figura 14, Figura 15, Figura 16.

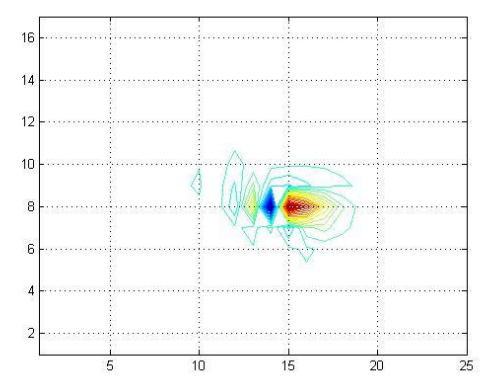

Figura 5: Transporte de Contaminante no lago Marajoara, com os parâmetros obtidos, calculados e apresentados neste estudo $t=1$. 


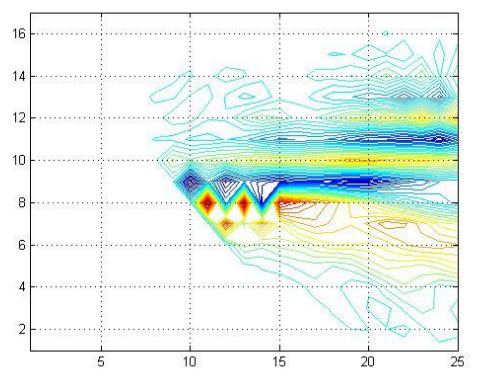

Figura 6: Transporte de Contaminante no lago Marajoara, com os parâmetros obtidos, calculados e apresentados neste estudo $t=10$.

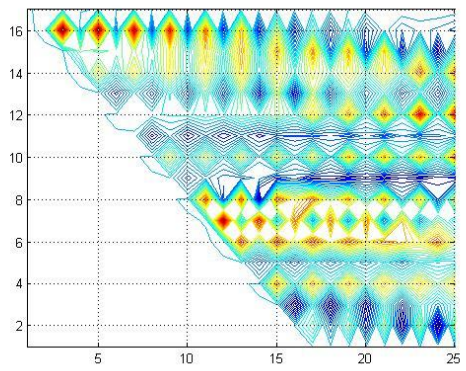

Figura 7: Transporte de Contaminante no lago Marajoara, com os parâmetros obtidos, calculados e apresentados neste estudo $t=40$.

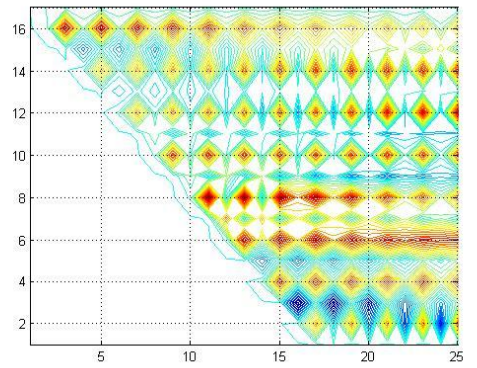

Figura 8: Transporte de Contaminante no lago Marajoara, com os parâmetros obtidos, calculados e apresentados neste estudo $t=120$.

Os resultados gerados pelo software Matlab mostram que poluente hipotético considerado neste modelo se dispersa rapidamente no meio aquático em uma direção preferencial devido a ação dos fenômenos climáticos. Nas Figuras 5, 6, 7, 8, 9, 10, 11, 12, 13, 14, 15 e 16 observa-se o espalhamento da pluma contaminante no lago em sua maior parte para a direita. De fato, este fenômeno ocorre em função da direção considerada para os ventos ser predominate para nordeste.

Como pode ser observado, quando $t=1$ (Figuras 5 e 11), a região com maior concentração de poluentes,

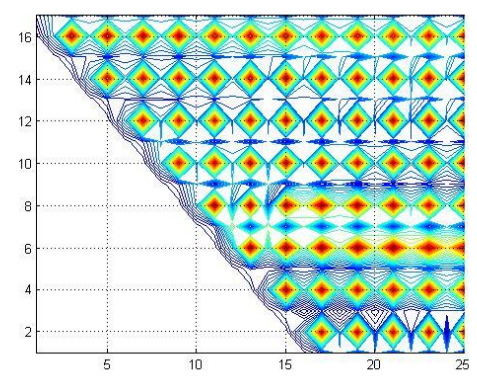

Figura 9: Transporte de Contaminante no lago Marajoara, com os parâmetros obtidos, calculados e apresentados neste estudo $t=480$.

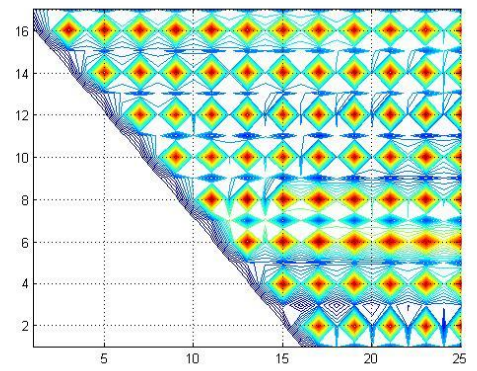

Figura 10: Transporte de Contaminante no lago Marajoara, com os parâmetros obtidos, calculados e apresentados neste estudo $t=1000$.

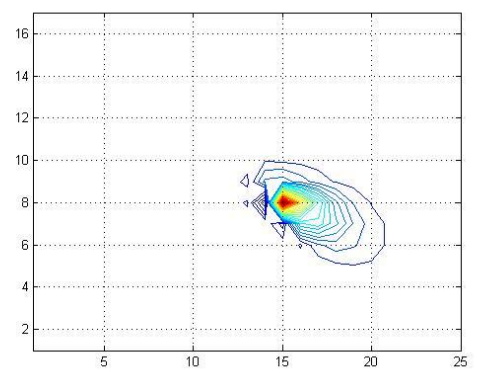

Figura 11: Transporte de Contaminante no lago Marajoara, com os parâmetros retirados de (Prestes et al., 2013) $t=1$.

representada pela pluma vermelha, está concentrada em uma pequena região. A medida que o tempo varia, o poluente se espalha no lago (Figuras 13, 14, 15 e 16), originando regiões com menor concentração do poluente, representadas pela cor azul.

Comparando as imagens geradas para ambas simulações em $t=40$ e $t=120$, é possível observar que nas Figuras 7 e Figura 8 o poluente encontra-se mais concentrado, com plumas vermelhas mais decorrentes, enquanto na Figura 13 e 14, a pluma se propaga com uma concentração menor. Tal fenômeno pode ser explicado 


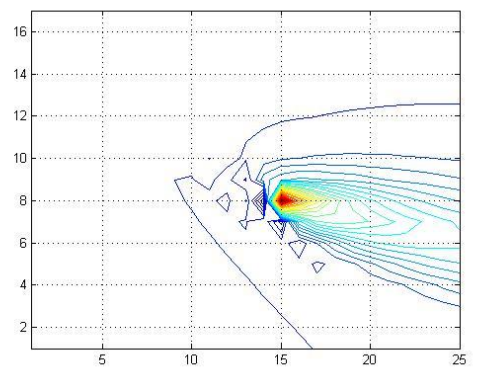

Figura 12: Transporte de Contaminante no lago Marajoara, com os parâmetros retirados de (Prestes et al., 2013) $t=10$.

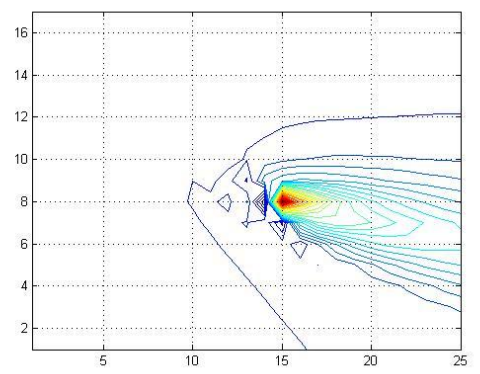

Figura 13: Transporte de Contaminante no lago Marajoara, com os parâmetros retirados de (Prestes et al., 2013) $t=40$

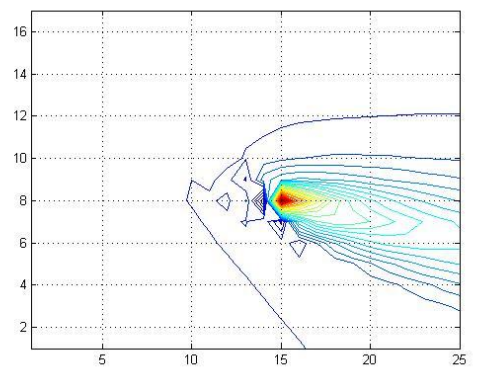

Figura 14: Transporte de Contaminante no lago Marajoara, com os parâmetros retirados de (Prestes et al., 2013) $t=120$.

pela alta taxa de difusibilidade utilizada na modelagem da dispersão do contaminante apresentada na Figura 13 , o que aumenta a velocidade com que o poluente se difunde em meio aquático.

Os resultados obtidos na simulação de um contaminante no lago Marajoara usando o modelo de adveçãodifusão, considerando tanto os parâmetros calculados e apresentados neste trabalho, quanto com os parâmetros retirados de (Prestes et al., 2013) são considerados resultados interessantes pois estão de acordo com os resultados esperados.

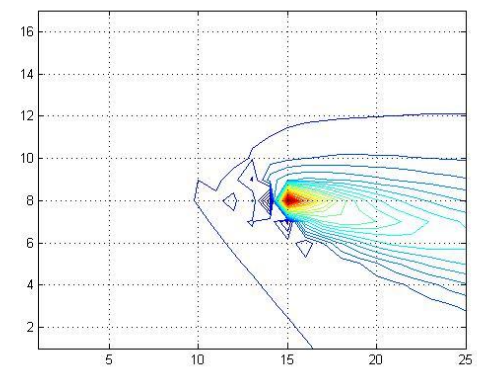

Figura 15: Transporte de Contaminante no lago Marajoara, com os parâmetros retirados de (Prestes et al., 2013) $t=480$

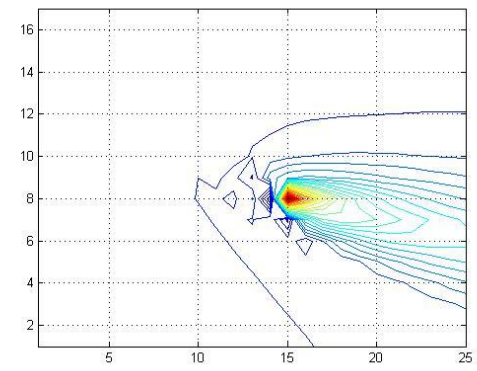

Figura 16: Transporte de Contaminante no lago Marajoara, com os parâmetros retirados de (Prestes et al., 2013) $t=1000$.

\section{Considerações Finais}

O lago do Marajoara é parte integrante de uma pequena praça que serve como sistema recreacional para a comunidade que vive nos bairros Marajoara e Philadelfia. Infelizmente, devido ao constante lançamento de poluentes no lago, o ambiente perdeu alguns de seus atrativos, recebendo a aparência de um local mal abandonado, cuidado e, em alguns pontos, mal cheiroso. Por ser um ambiente de socialização entre os habitantes da região e por estar localizado à proximidade de uma região hospitalar, se faz necessário um estudo detalhado da influência de contaminantes no lago e no meio ambiente, de forma que possa auxiliar à intervenção de órgãos públicos para revitalizar o local e tornar o ambiente mais agradável, tratando a água do lago e harmonizando-o com a paisagem. No sentido qualitativo, é fácil perceber que a água está poluída, mas não é feito medições adequadas para fornecer avaliações mais quantitativas que oferecerão os dados necessários à elaboração de projetos. Assim, simulações são importantes, pois oferecem esses dados e dão uma ideia de como o poluente se comporta, ajudando a desenvolver a melhor forma de combatê-lo. 
A análise da dispersão de contaminantes em meio aquático, por meio do modelo Difusão-Advecção, permite um melhor entendimento sobre como a contaminação em apenas uma fonte pontual pode influenciar toda a região de um lago.

Constata-se que usando a constante de difusibilidade menor, o poluente difunde lentamente no meio aquático.

Vale destacar a importância de softwares como o Matlab que auxiliam para uma melhor compreensão e visualização do comportamento da dispersão de poluentes e que, em função dos fenômenos naturais da região, é fundamental que haja tratamento adequado para evitar maiores danos ao meio ambiente.

\section{Referências}

Abril, R. (2014). Como é possível recuperar um rio poluído. URL <http: //mundoestranho.abril.com.br/materia/ como-e-possivel-recuperar-um-rio-poluido>.

Bassanezi, R. (2002). Ensino-aprendizagem com modelagem matemática: uma nova estratégia. Contexto, URL https: //books. google. com. br/books?id=4VDcgy296cMC.

Danconi, L. A., Poletti, E. C. C., Angelis, A. F. (2013). Modelagem e simulaç ao numérica da dispersão de poluentes via equação de difusão -advecção. Congresso de Matemática Aplicada e Computacional - CMAC Sudeste.

Fleck, L., Tavares, M. H. F., Eyng, E. (2013). Especifidades e importância de modelos matemáticos de qualidade da água. Revista EIXO, 2(1), 106-119.

Fortuna, A. d. O. (2000). Técnicas Computacionais para Dinâmica dos Fluidos: Conceitos Básicos e Aplicações, $1^{\circ}$ edn. Editora da Universidade de São Paulo.

INMET (2015). Instituto nacional de meteorologia. URL <http://ww . inmet.gov.br/portal/index.php?r= estacoes/estacoesAutomaticas $>$.

Júnior, G. B. L., Schulz, H. E. (2012). Análise de condições de contorno para a quantificação da transfêrencia de massa unidimendional em regime turbulento. Em: XXXIV Congresso Nacional de Matemática Aplicada e Coputacional.

Lopes, A. A. S., Souza, L. C., Gomes, J. L., Silva, J. M. (2014). Modelagem da dispersão de material impactante em águas rasas via equações de difusão -advecção. $2^{\circ}$ Workshop de Integração UFVJM.

Poletti, E. C. C. (2009). Dispersão de poluente em sistema de reservatório: Modelagem matemática, e simulação computacional utilizando aproximação numérica e conjuntos fuzzy. Tese de Doutorado, Universidade Estadual de Campinas.
Prestes, M. F. B., ao Frederico C. A. Meyer, J., Poletti, E. C. C. (2013). Dispersão de material impactante em meio aquático: modelo matemático, aproximação numérica e simulação computacional - reservatório do salto grande, americana-sp. Biomatemática, 23(1), 4356.

UNRIC (2014). Relatório da onu mostra população mundial cada vez mais urbanizada, mais de metade vive em zonas urbanas ao que se podem juntar 2,5 mil milhões em 2050. URL <http://ww.unric.org/pt/actualidade/ 31537-relatorio-da-onu-mostra-populacao-mundial>.

USP (2014). Revista eletrônica de ciência. URL <http://www.cdcc.sc.usp.br/ciencia/artigos/ art_32/atualidades.html $>$.

Vargas, B. M., da S. Freire, P. C., Accetta, D., Uhrenholdt, T., Amorim, J. C. C. (2001). Estudo de circulação d'água e de dispersão de poluentes no complexo lagunar sul de santa catarina. Em: XIV Simpósio Brasileiro de Recursos Hídricos. 